\title{
Chemotaxonomy: An Approach for Conservation \& Exploration of Industrially Potential Medicinal Plants
}

\section{Ankita Misra* and Sharad Srivastava}

Pharmacognosy Division, CSIR-National Botanical Research Institute, Lucknow, India

\section{Editorial}

The use of medicinal plants for the benefit of mankind is timeless and is undoubtedly increasing day by day and there their taxonomic categorization is essential for the better understanding of plant community, their origin and association with each other. In our nature, plant ecosystem consists of several biotic elements like angiosperm, fungi, mosses, lichens, algae and they are rich source of natural products, varied hugely in chemical constituents. Among them, angiosperms are the most developed and plays very crucial role in benefit of mankind for healthcare. More effectively medicinal plants in particular, have huge importance in present scenario of health care industry which is concurrently moving towards safe and effective alternatives.

The plants are classified on the basis of morphological, anatomical and chemotaxonomic classification. Among these systems of species characterization first two are traditional one, whilst the third system is comparatively a newer approach. The chemotaxonomy emerged from; chemo+ taxonomy, which itself explains as "the classification of plant species on basis of their chemical constituents. The concept of chemotaxonomy has existence in past centuries and evidence of variation in metabolic profile was utilized for classification purposes by folk taxonomist. Although some other morphological/anatomical factors were also taken into the consideration viz. edibility, taste, color, smell without having much information about chemical properties. It is interesting to observe that, there is increasing fondness of researcher's towards chemotaxonomy which is due to the peculiar nature and structure of the basic chemical moiety of secondary metabolites. As the secondary metabolites are often specific and particular to taxonomically related species and thus helps as efficient tool for classification. However, one must have sound knowledge about the biosynthetic pathway(s) of secondary metabolites in plant for accurate categorization. This amalgamation of analyzing chemical profiling of targeted species with the morphological characters leads to edge cutting tool for their characterization and classification. In addition, it is also feasible for researchers to determine the evolution and phylogeny of particular species or plant family with its chemotaxonomic profile. One other significant contribution of chemo taxonomy is providing a rationale study for identification and quantification of specific class of natural compound/chemical moiety in particular taxon and hence became advantageous in further study of unexplored species lying within same genera/taxon.

Studies which support the phyto-geographical abundance, genetic/ molecular data and morpho-anatomic characteristics are still sparingly available for some genera [1] and thus addition of chemotaxonomic aspect to existing knowledge will provide a more significant/wide phylogenetic distribution, due to presence of secondary metabolites which are characteristically synthesized in taxon [2]. Moreover, as the primary metabolites are ubiquitously distributed, they are not effective target for taxonomical distribution of plant species. Thus, the distribution pattern of secondary metabolites in plant species will bring new information for the taxonomic classification. The methods used for chemotaxonomic classification is much better and advanced than classical one and aids in modern day studies of natural product development, either it is procurement and, processing of plant material or quantitative \& qualitative estimation of metabolite. The upliftment of advances in analytical chemistry with the development of sophisticated methods viz. HPLC, UPLC, OPLC etc. in qualitative and quantitative estimation of chemical compounds up to trace amount of ug and/or ng leads to rise and strengthening of chemotaxonomy as a branch of plant classification [3]. This has become evident from some recently floating studies on chemotaxonomic studies on some potential plant families in various genera viz. Malvaceae, Ranunculaceae, Magnoliaceae, Polygonaceae, and Solanaceae [4].

Indeed it is noteworthy to mention that the chemotaxonomic studies not only serve for classification purposes, but also it can be extended further for benefit of humans in exploration of commercially viable and therapeutically important medicinal plants. Several studies have been reported for chemotaxonomic characterization of genera and families for better understanding of potential species viz. Cannabis, Acacia, Withania, Ornithine derivative alkaloids and sometime also as a tool for authentication of herbal drug i.e., Chamomile etc. In addition to this, introduction of chemotaxonomy for identification of intra-specific variation in terms of secondary metabolites lead to very promising results [5-7]. This includes qualitative and quantitative estimation of metabolites within the species, targeting the wild population from their natural habitat. Chemotaxonomic studies will guide researchers' toward the identification of high metabolite yielding germplasm, due to several factors viz. genetic, epigenetic, environmental etc. Identification of potential germplasm and with the use of technological advancement the production of therapeutically viable metabolites can be harnessed for commercial/industrial use. Moreover this also aids in site specific exploration of high metabolite yielding chemotype to meet out the commercial demands and, supply and to promote the agriculture practices of these industrial crop for societal upliftment in area having similar phytogeographical locations.

\section{References}

1. Bremer B (2009) A review of molecular phylogenetic studies of Rubiaceae 1 Ann Mo Bot Gard 96: 4-26.

2. Wink M (2003) Evolution of secondary metabolites from an ecological and molecular phylogenetic perspective. Phytochemistry 64: 3-19.

*Corresponding author: Ankita Misra, Pharmacognosy Division, CSIR-National Botanical Research Institute, Lucknow, India, Tel: 0522-2297818; E-mail anku.mis@gmail.com

Received September 30, 2016; Accepted November 14, 2016; Published November 18, 2016

Citation: Misra A, Srivastava S (2016) Chemotaxonomy: An Approach for Conservation \& Exploration of Industrially Potential Medicinal Plants. J Pharmacogn Nat Prod 2: e108. doi: 10.4172/2472-0992.1000e108

Copyright: ( $) 2016$ Misra A, et al. This is an open-access article distributed unde the terms of the Creative Commons Attribution License, which permits unrestricted use, distribution, and reproduction in any medium, provided the original author and source are credited. 
Citation: Misra A, Srivastava S (2016) Chemotaxonomy: An Approach for Conservation \& Exploration of Industrially Potential Medicinal Plants. J Pharmacogn Nat Prod 2: e108. doi: 10.4172/2472-0992.1000e108

Page 2 of 2

3. Bhargava VV, Patel SC, Desai KS (2013) Importance of terpenoids and essential oils in chemotaxonomic approach. Int J Herbal Med 1: 14-21.

4. Sivarajan VV (1991) Introduction to the Principles of Plant Taxonomy. Cambridge University Press.

5. Srivastava S, Misra A, Shukla PK, Kumar B, Lata S, et al. (2014) A validated over pressured layered chromatography (OPLC) method for separation and quantification of colchicine in Gloriosa superba (L.) tubers from different geographical regions. RSC Adv 4: 60902.
6. Misra A, Srivastava S, Srivastava P, Shukla P, Agrawal PK, et al. (2016) Chemotaxonomic variation in forskolin content and its correlation with ecogeographical factors in natural habitat of Coleus forskohlii Briq. collected from Vidarbha (Maharashtra, India). Indus Crops Prod 84: 50-58.

7. Shukla PK, Misra A, Kumar M, Rajan S, Agrawal PK, et al. (2016) Intra-Specific Chemotypic Variability of Forskolin Content in Coleus forskohlii (Wild.) Briq Growing in Nilgiri Hills of India. JPC 29: 347-355. 